\title{
In adolescence, extreme prematurity is associated with significant changes in the microvasculature, elevated blood pressure and increased carotid intima-media thickness
}

\author{
HaNa Lee, ${ }^{1}$ Sabrina Dichtl, ${ }^{1}$ Zuzanna Mormanova, ${ }^{1}$ Robert Dalla Pozza, ${ }^{2}$ \\ Orsolya Genzel-Boroviczeny ${ }^{1}$
}

${ }^{1}$ Division of Neonatology IS, Hauner Dr. von Hauner Children's Hospital, University of Munich, Munich, Germany ${ }^{2}$ Department of Pediatric Cardiology, Hauner Dr. von Hauner Children's Hospital, University of Munich, Munich, Germany

\section{Correspondence to} Professor Orsolya GenzelBoroviczény, Division of Neonatology IS, Hauner University Children's Hospital, University of Munich, Maistr. 11, Munich 80337, Germany; Genzel@med.Imu.de

$\mathrm{HL}$ and SE contributed equally.

Received 14 March 2013 Revised 26 April 2014 Accepted 12 May 2014 Published Online First 30 May 2014
CrossMark

To cite: Lee HN, Dichtl S, Mormanova Z, et al. Arch Dis Child 2014:99: 907-911.

\begin{abstract}
Objectives Increased carotid intima-media thickness (cIMT) and loss of capillary density are antecedents of cardiovascular disease in adults. Former preterm infants are at risk for metabolic precursors of vascular disease, but vascular changes have not yet been studied in this population.
\end{abstract}

Patients and methods In 54 former preterm (birth weigh (BW) $753 \pm 152 \mathrm{~g}$ ) and 12 term children (BW $3395 \pm 558 \mathrm{~g}$ ), functional vessel density (FVD) on the forearm and cIMT were obtained at a mean $( \pm \mathrm{SD})$ age of $11.8 \pm 1.5$ years. FVD was measured using sidestream dark field imaging before, during and after arterial occlusion, and cIMT using high resolution B-mode ultrasonography. Statistical comparisons were made using the $\mathrm{t}$ test, Mann-Whitney $\mathrm{U}$ test and the sign test for symmetry, with a two-sided $p$ value as appropriate.

Results The systolic blood pressure of the former preterm children was significantly increased compared to reference values (females $119 \pm 8, z$ score 1.23; males $118 \pm 11, z$ score $1.14 \mathrm{~mm} \mathrm{Hg}$ ), as were the cIMT values $(0.45 \pm 0.03 \mathrm{~mm}$ vs $0.38 \pm 0.04 \mathrm{~mm} ; p<0.001)$. FVD was significantly higher in former preterm children compared to controls at baseline (mean $\pm S D 33.6 \pm 14.5 \mathrm{~cm} / \mathrm{cm}^{2}$ vs $23.0 \pm 7.1 \mathrm{~cm} / \mathrm{cm}^{2} ; p=0.002$ ) with decreased reactivity during arterial occlusion $\left(\chi^{2} 7.14, p=0.008\right)$.

Conclusions Preterm birth is associated with significant permanent alteration in the microcirculation and increased cIMT and systolic blood pressure. It is unknown whether these changes are due to preterm birth and rapid maturation of the skin or to nutritional factors, as previously thought. This is an important area of future research.

\section{INTRODUCTION}

Low birth weight due to intrauterine growth restriction (IUGR) and/or preterm birth has been reported to be associated with an increased prevalence of high blood pressure, arterial stiffness and endothelial dysfunction, high blood pressure, dyslipidemia, insulin resistance and obesity. ${ }^{1-7}$ The association of low birth weight and cardiovascular risk may be due to imprinting during a vulnerable age. ${ }^{8}$

Carotid intima-media thickness (cIMT) is an indicator of generalised atherosclerosis and strongly associated with future cardiovascular events. Increased cIMT correlates with most cardiovascular risk factors, such as high blood pressure and diabetes. ${ }^{10-13}$ Recently, normative cIMT values for

\section{What is already known on this topic?}

- Low birth weight is a known risk factor for metabolic disease.

- Increased blood pressure, glucose intolerance and obesity seem more common in former preterm infants, which might put them at risk for cardiovascular disease as adults.

\section{What this study adds?}

- As adolescents, former preterm infants have increased carotid intima-media thickness and presumably permanent changes in the skin microcirculation.

- This increases concern regarding their cardiovascular health as adults.

children and adolescents have been published, but there are no data on the cIMT of former preterm children. ${ }^{14}$ Impaired microcirculation with capillary rarefaction of the nail fold is associated with hypertension and might precede the development of hypertension. ${ }^{15} 16$

The primary aim of our study was to compare cIMT, blood pressure and skin capillary density before, during and after arterial occlusion in former very preterm children relative to reference values.

\section{METHODS}

Subjects

The families of children with a birth weight of $<1000$ g born between 1995 and 1999 at our institution were contacted in 2009 when the children were 9-14 years old. We previously reported detailed data on the postnatal nutritional support and growth of this cohort. ${ }^{17}$ Healthy children not taking any medications were recruited from a tertiary healthcare centre (University Children's Hospital Munich) and from a nearby elementary school as controls. The parents or legal guardians of all participants and the children themselves gave written informed consent and the local ethics committee approved the study protocol and consent forms. 


\section{Measurements}

Weight, height, waist and hip circumference were measured with the same equipment for all children according to a standardised protocol. Height was measured with a fixed and calibrated anthropometer and weight with a calibrated bioelectrical impedance analysis scale using electrical resistance. A flexible, non-elastic measuring tape was used to determine waist and hip circumference. Results were compared with German normative values, the Kromeyer-Hauschild percentiles. ${ }^{18}$

Blood pressure was measured in triplicate on the right arm with an automated blood pressure meter (DINAMAP Pro 100) and appropriate cuff size to guarantee precise values. The mean of the triplicate values was used for comparisons. Subjects were in sitting position with the arm at heart level. Data from the Update on the 1987 Task Force Report on High Blood Pressure in Children and Adolescents were used as normative values ${ }^{19}$ and differences from the blood pressure of the subjects given as z scores. $\mathrm{z}$ Scores allow calculation of the probability of a score occurring within a normal distribution and enable the comparison of two scores from different normal distributions.

The ultrasonographic study was performed according to a standardised scanning protocol with the subject in a supine position in a quiet room at $22^{\circ} \mathrm{C}$ as previously reported. ${ }^{12}$ The cIMT was defined as the distance between the leading edges of the lumen-intima and the media-adventitia interface of the far wall. Two measurements on each side were taken by one of three experienced sonographers and stored digitally for off-line analysis. The investigators carrying out the ultrasound were blinded to the birth weight and gestational age of the subjects, but did know that the children were participating in a clinical study, although they were not informed about the details of the study. The computer software (QLAB; Philips Medizin Systeme) analysed the cIMT at 64 different points within the selected $10 \mathrm{~mm}$ segment. The calculated mean of four measurements, two separate scans on each side, provided the arithmetic mean cIMT of each subject (a total of 256 points analysed). The intraobserver and interobserver coefficient was measured in a previous study. ${ }^{12}$ Due to scheduling problems seven children were unable to participate in the cIMT measurements. Their basic and follow-up data did not differ from those of the other 47 children.

\section{Sidestream dark field imaging}

The sidestream dark field (SDF) device is a hand-held microscope used to observe the microcirculation non-invasively, and consists of a central light guide that is surrounded by concentrically placed green light-emitting diodes (LEDs). The light (wavelength $530 \mathrm{~nm}$ ) is at the optimal wavelength for absorption by the deoxy- and oxyhaemoglobin of red blood cells. SDF imaging is validated by comparison with orthogonal polarisation spectral imaging and intravital microscopy. ${ }^{20}$

All children were seated in a comfortable and stable position with their arms bent slightly at heart level. Sodium chloride $0.9 \%$ was applied on the first quarter of the right forearm to ensure more translucent skin. The device held firmly in a special holder was adjusted for optimal focus and contrast. Images were recorded before, during and after arterial occlusion for a total of $300 \mathrm{~s}$ to assess the response of vessel density to occlusion and release. Arterial occlusion was produced by a cuff on the upper arm (cuff pressure $>$ systolic blood pressure). Images were stored directly on the hard disk of a laptop and underwent blinded off-line analysis with Microvision Analysis Software (MAS), a semi-automatic analysis software which automatically detects vessels on the screen and allows individual corrections for artefacts, for example, hair. The programme subsequently calculates the functional vessel density (FVD), which represents total perfused vessel length in $\mathrm{cm}$ per image area in $\mathrm{cm}^{2}$ and the distribution of vessel diameter, which gives the distribution of small $<10 \mu \mathrm{m}$, medium $10-20 \mu \mathrm{m}$ and large $20-100 \mu \mathrm{m}$ vessels as a percentage of the total vessel length.

Most former preterm children indicated on the reply form to the invitation to participate, that they would decline if any laboratory work was planned. Therefore only non-invasive measurements were carried out.

\section{Statistics}

The sign test for symmetry was used to compare the clinical data of the subjects, such as body mass index (BMI), hip-waist ratio and blood pressure, to normative values. ANOVA for repeated measurements and non-parametric data (Friedman test) followed by Tukey's post hoc test for parametric or Dunn's post hoc test for non-parametric data were used to assess changes in parameters within a group. For comparison before and after arterial occlusion within the groups, the Wilcoxon signed rank test for non-parametric data and a between-group nonparametric Mann-Whitney U test were performed with adjustment for multiple comparisons. To assess the time to the minimum vessel density during arterial occlusion, the log-rank test was used. Correlation was tested by Spearman's rank correlation test. Differences between the patients' cIMT and normative values from the literature were tested using the one-sample t test. A two-sided $\mathrm{p}$ value $<0.05$ was considered significant. Results are presented as mean $\pm \mathrm{SD}$. All analyses were performed with GraphPad Prism 5.0 for Windows Vista (GraphPad Software, San Diego, California, USA). z Scores show whether an observation is equal ( $\mathrm{z}$ score -0.99 to 0.99 ), below $(\leq-1)$ or above $(\geq 1)$ the mean of the normative values. A $z$ score $\geq 1.645$ is statistically significant at the 0.05 level.

\section{RESULTS}

\section{Subjects}

Of the 166 infants in the original cohort, we were able to trace 77 families and 54 children agreed to participate in our follow-up study at 10-14 years of age. Children lost to follow up or declining participation did not differ from the participants in birth weight, gestational or postnatal growth until discharge (mean \pm SD perinatal data from the current study: birth weight $765 \pm 156 \mathrm{~g}$; gestational age $26.4 \pm 1.9$ weeks; birth weight regained at $14.8 \pm 5.7$ days; weight gain $/ \mathrm{kg}$ body weight/day after birth weight was regained $13.5 \pm 2.8 \mathrm{~g}$; time to reach full enteral feedings $23.7 \pm 9.9$ days; protein intake $/ \mathrm{kg} /$ day $<3 \mathrm{~g} 11.0$ \pm 5.3 days; caloric intake $>100 \mathrm{kcal} / \mathrm{kg} /$ day $10.0 \pm 5.1$ days).

The data of the former extremely low birthweight (ELBW) children (ex-ELBW) were also compared between sexes and for small for gestational age (SGA) versus appropriate for gestational age (AGA) children (table 1). The weight of the male SGA children was significantly reduced in comparison to male AGA children and height was compromised in female SGA children compared to female AGA children. BMI calculated as percentile for age varied widely but was low for the majority of children, with no significant difference between the sexes or between AGA and SGA. However, stratifying for AGA/SGA and sex did result in very small groups.

All ex-ELBW children had a significantly higher waist-hip ratio than the reference population $(p<0.001)$, but for different reasons. Hip circumference was significantly reduced in SGA children of both sexes, while waist circumferences were within 
Table 1 Clinical data of the subjects

\begin{tabular}{|c|c|c|c|c|c|c|}
\hline & \multicolumn{4}{|c|}{ Ex-ELBW $(n=54)$} & & \\
\hline & \multicolumn{2}{|l|}{ Male $(n=24)$} & \multicolumn{2}{|c|}{ Female $(n=30)$} & \multicolumn{2}{|c|}{ Control $(n=12)$} \\
\hline & SGA $(n=11)$ & AGA $(n=13)$ & SGA $(n=9)$ & AGA $(n=21)$ & Male $(n=7)$ & Female $(n=5)$ \\
\hline Gestational age (weeks) & $28(2)$ & $25.9(1.6)$ & $27.5(2.7)$ & $26.4(1.8)$ & $39.6(1.4)$ & $39.2(1.1)$ \\
\hline Birth weight (g) & 686 (184) & $804(94)$ & $643(174)$ & 803 (123) & $3569(466)$ & $3070(558)$ \\
\hline Birth length $(\mathrm{cm})$ & $30.8(3.2)$ & $33.4(2.2)$ & $31.9(4)$ & $32.9(2.7)$ & $53.3(1.7)$ & $48.8(5.9)$ \\
\hline \multicolumn{7}{|l|}{ APGAR values at } \\
\hline $1 \mathrm{~min}$ & $4.9(2.8)$ & $4.2(2.7)$ & $5.3(2.2)$ & $5.5(2.4)$ & & \\
\hline $5 \mathrm{~min}$ & $7.1(1.7)$ & $6.5(1.7)$ & $7.4(1.8)$ & $7.6(1.8)$ & & \\
\hline $10 \min$ & $7.7(1.2)$ & $7.4(1.4)$ & $8.0(1.7)$ & $8.2(1.4)$ & & \\
\hline Umbilical pH & $7.29(0.05)$ & $7.21(0.08)$ & $7.3(0.05)$ & $7.3(0.05)$ & & \\
\hline \multicolumn{7}{|l|}{ Current study } \\
\hline Age (years) & $11.4(1.6)$ & $11.8(1.3)$ & $11.3(1.6)$ & $11.9(1.7)$ & $10.2(0.8)$ & $10.7(1.4)$ \\
\hline Weight (kg) & $31.6(6)^{*}$ & $41(11)$ & $33.6(15)$ & 39.5 (8.6) & 37 (10.6) & $36.6(4)$ \\
\hline Height $(\mathrm{cm})$ & $142(11)$ & $147(12)$ & $145(14)$ & $150(11)$ & & \\
\hline BMI & $17.6(3)$ & $18.5(3)$ & $16.8(3)$ & $16.7(4)$ & & \\
\hline \multirow[t]{2}{*}{ Waist $(\mathrm{cm})$} & $62(6)$ & $69(11)$ & $64(12)$ & $67(7)$ & & \\
\hline & $p=0.23$ & $p=0.02^{*}$ & $p=1$ & $p=0.03^{*}$ & & \\
\hline \multirow[t]{2}{*}{ Hips (cm) } & $70(5)$ & $77(9)$ & $72(13)$ & $78(7)$ & & \\
\hline & $p=0.01 \dagger$ & $p=0.58$ & $p=0.04 \dagger$ & $p=0.19$ & & \\
\hline \multirow[t]{2}{*}{ Waist-hip ratio } & $0.88(0.03)$ & $0.89(0.05)$ & $0.88(0.02)$ & $0.86(0.05)$ & & \\
\hline & $p<0.001$ * & $\mathrm{p}<0.001$ * & $\mathrm{p}<0.001$ * & $\mathrm{p}<0.001$ * & & \\
\hline BP systolic & $110(11)$ & $125(10)$ & $116(8)$ & $121(8)$ & & \\
\hline z Score & 0.44 & $1.73 \ddagger$ & 1.04 & 1.31 & & \\
\hline BP diastolic & $63(5)$ & $69(6)$ & $66(5)$ & 68 (5) & & \\
\hline z Score & -0.17 & 0.4 & 0.1 & 0.22 & & \\
\hline
\end{tabular}

the norm, whereas AGA children had normal hip circumferences but significant higher waist circumferences. ${ }^{18}$

Systolic blood pressure was significantly increased in the ex-ELBW children compared to normative values for children of The National Heart, Lung, and Blood Institute, Maryland (table 1). ${ }^{19}$ AGA children had higher systolic blood pressure values compared to SGA infants $(112 \pm 8.2 \mathrm{~mm} \mathrm{Hg}$ vs 122

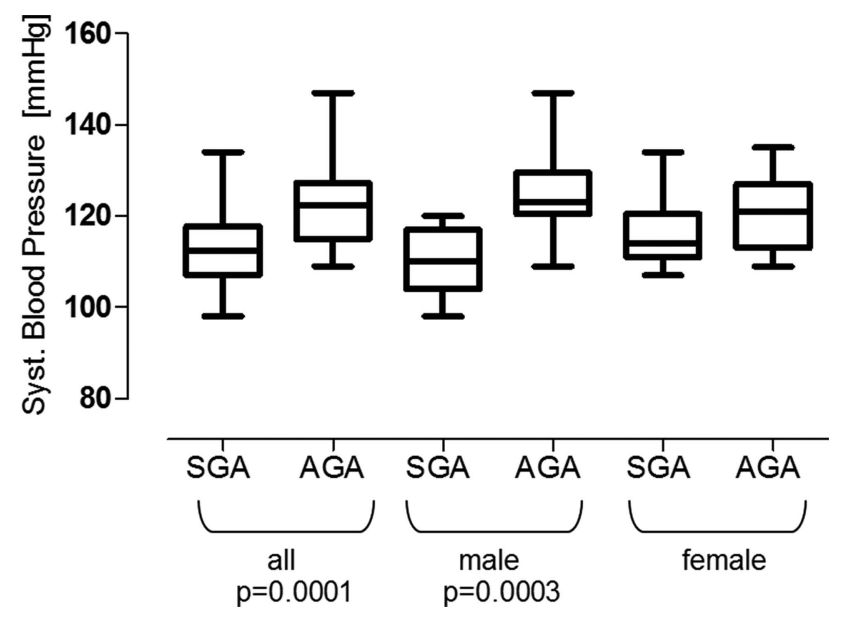

Figure 1 Systolic (Syst.) blood pressure of former preterm infants when adolescents, small for gestational age (SGA) versus appropriate for gestational age (AGA) birth weights. $\pm 8.5 \mathrm{~mm} \mathrm{Hg} ; \mathrm{p}=0.0001)$. This difference is mostly the result of the higher blood pressure of the AGA boys, with only a trend but no significant difference in girls (figure 1). As an entire group, males and females did not differ significantly $(p=0.64)$. Diastolic blood pressure did not differ significantly.

cIMT was significantly increased in our former ELBW children $(0.45 \pm 0.03 \mathrm{~mm})$ compared to the age and gender-specific normative value $(0.38 \pm 0.04 \mathrm{~mm} ; \mathrm{p}<0.0001)$ given in the literature. ${ }^{14}$ There was no correlation between cIMT values and systolic blood pressure $(r=0.2, p=0.18)$. cIMT did not differ between sexes or between AGA and SGA.

\section{Microcirculation}

Ex-preterm children had significantly higher FVD at rest (ex-ELBW: $33.6 \pm 14.5 \mathrm{~cm} / \mathrm{cm}^{2}$ vs control: $23.0 \pm 7.1 \mathrm{~cm} / \mathrm{cm}^{2}$; $\mathrm{p}=0.002)$ during arterial occlusion and after release of occlusion (figure 2). Median time to reach the minimum FVD after arterial occlusion was significantly longer for the preterm group than for the control group ( $30 \mathrm{~s}$ vs $10 \mathrm{~s}, \chi^{2} 7.14, \mathrm{p}=0.008$, ratio $1.5,95 \%$ CI 0.9 to 2.1 ). During reactive hyperaemia, FVD increased significantly in both groups (preterm group $\mathrm{p}=0.002$ vs control group $\mathrm{p}=0.04$ ) and was followed by a decrease to their respective minimums, which the preterm group reached significantly faster (within $120 \mathrm{~s}$ vs $180 \mathrm{~s}$ for the control group; $\mathrm{p}=0.03$ ). FVD increased continuously in both groups up to the initial baseline values. The relative distribution in percentage of vessel diameters (small, $<10 \mu \mathrm{m}$ and median, 10-20 $\mu \mathrm{m}$ ) was not significantly different between the two groups at any time 


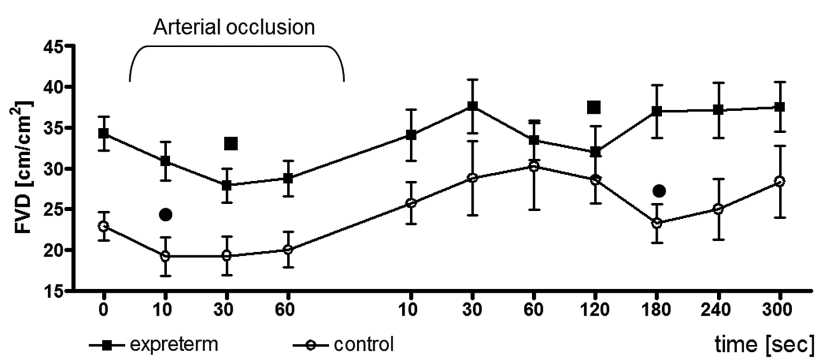

Figure 2 Skin capillary density (functional vessel density, FVD) before, during and after arterial occlusion of former preterm (ex-preterm $n=54$ ) and term children (control, $n=17)$. The difference in FVD between the two groups is statistically significant at all times points. Minimum is indicated by $\mathbf{m}$ in the ex-preterm children and by in the control group.

nor did it change significantly within the groups during arterial occlusion and release. There were no large vessels with diameters $>20 \mu \mathrm{m}$. No correlation was found between capillary density and systolic blood pressure values. FVD did not differ between sexes or between SGA and AGA.

\section{DISCUSSION}

Our study found that adolescent former very preterm infants have significant changes in macro- and microcirculatory parameters with increased systolic blood pressure and intima-media thickness, higher capillary density and altered reaction to arterial occlusion.

So far microvascular rarefaction, not increased capillary density, has been linked to hypertension, both as a result and a cause. $^{21}$ Capillary rarefaction has also been demonstrated in former preterm infants before venous occlusion, but a previous similar study with more mature preterm infants showed no difference in capillary density between former preterm and term children. ${ }^{42}$ This discrepancy with the finding of higher capillary density in the skin of our ex-preterm group is most likely due to the better image quality of our device. All previous studies of the microcirculation in former preterm infants have used capillary microscopy of the nail fold. Capillary microscopy and orthogonal polarisation spectral imaging provide very similar results for vessel diameter and red blood cell (RBC) velocity in the nail bed, ${ }^{21}$ but SDF imaging has a significantly higher image quality with more detail, capillary contrast and quality, and granularity of venular RBC columns, and less motion blur due to the use of stroboscopic LED ring-based SDF illumination. This optical improvement made it possible to obtain good images from the distal forearm in our subjects, where mostly very small vessels with diameters $<10 \mu \mathrm{m}$ were seen in both groups (71\% and $75 \%$, respectively) with only a small proportion of medium-sized vessels with diameters of 10$20 \mu \mathrm{m}$ and no larger vessels. Therefore, capillary rarefaction could be restricted to the larger capillaries not seen in the skin but only in the nail fold. In a previous study on preterm infants, we have shown that capillary diameter changes significantly with persistence of an open ductus arteriosus. ${ }^{23}$ Thus, the differing findings in microcirculation studies are possibly due to different methods and different capillary beds. This has been shown for laser Doppler imaging and nail fold microscopy in adults with systemic sclerosis. ${ }^{24}$ SDF imaging is a newly developed method with better contrast and resolution and allows the investigation of capillaries in a different layer than other devices. It is possible that capillary rarefaction of vessels
$>20 \mu \mathrm{m}$ is compensated for by an increase in smaller vessels, as we have seen in preterm infants with an open ductus arteriosus. $^{23}$

No studies on changes in the microcirculation have reported detailed information about the early postnatal nutrition of their subjects. The intention of our original first study was see if increased protein intake would lead to growth rates comparable to in utero growth. Our infants showed increased growth but did not achieve intra-uterine growth rates. Postnatal growth is related to IGF-1 and to vessel formation, at least in the retina. More than a decade later we know that a protein intake of $3 \mathrm{~g} / \mathrm{kg} / \mathrm{day}$, which was usually achieved after 10 days, is not sufficient and the recommendations now are of up to $4 \mathrm{~g} / \mathrm{kg} / \mathrm{day}$ as soon as possible after birth. ${ }^{25}$ On the other hand, in term infants increased protein content in formula leads to a higher risk of obesity. ${ }^{26} \mathrm{We}$ did not record the post-discharge feeding of our subjects, but since they were still growth restricted at term, most of them received enriched formula or supplemented breast milk for some months. The demand for increased protein supplementation in preterm infants is driven by the knowledge that more protein leads to better brain growth and neurodevelopment. Our infants most likely received little protein during their hospital stay and possibly too much after reaching term. Both factors might have far-reaching consequences regarding vessel growth and maturation.

The increased cIMT of the former preterm infants is one of the earliest signs of atheroma formation. Finken et $a l^{27}$ also found an increased cIMT in adolescents born very preterm and felt that this was due more to their body composition at the time of measurement than to fetal or early and late postnatal growth patterns. Higher BMIs resulted in a worse lipid profile and thus led to higher cIMT values. Similarly to Finken et al, we also found an increased waist-to-hip ratio, which was due to a higher proportion of abdominal fat. This body fat distribution influences fat metabolism, since abdominal fat secretes free fatty acids and cytokines, which result in atheroma formation and intimal thickening. ${ }^{21}$ Increased cIMT thickness is also found in adolescents with hypertension, diabetes mellitus, hypercholesterolaemia and early enriched formula feeding for rapid catch-up growth in preterm infants. ${ }^{10}$ As mentioned earlier, a lower protein content in formula reduced obesity risk in term infants. $^{28}$

Our findings agree with previous studies that ex-preterm children have higher systolic blood pressure than term controls. ${ }^{4}$ In our study the former AGA preterm infants had significantly higher systolic blood pressure than the SGA as a group, but this was due to the difference in blood pressure only in male AGA and SGA children. Males do worse as preterm infants, possibly since the larger fetal growth trajectory makes them more vulnerable to insults. Males who are small at birth might escape some of these risks.

Different challenges can result in similar offspring outcome phenotypes in animal models. IUGR due to disruption of placental blood flow, maternal chronic hypoxia, glucocorticoid exposure, environmental insults such as stress, tobacco or endocrine-disrupting chemicals, restrictions in maternal diet and overnutrition result in very similar phenotypes that include obesity, hypertension, insulin resistance, type 2 diabetes and cardiovascular disease in the offspring, suggesting common or shared mechanistic pathways. ${ }^{29}$

Limitations of the study and possible bias are the response and willingness of parents and children to participate, although perinatal data were not different between included children and non-responders or decliners. 
Due to the time consuming nature of microcirculation measurements which also depend on the patience and cooperation of participants, it was difficult to obtain a larger number of microcirculation measurements in healthy children. This might have masked some possible significant differences between former term and preterm infants. It takes quite a long time to obtain microcirculation images without pressure or movement artefacts and to perform the off-line analysis.

This is the first study reporting detailed microcirculatory changes in former preterm infants, including changes in true capillaries with a diameter below $10 \mu \mathrm{m}$.

In conclusion, preterm birth poses a risk for cardiovascular health and seems to permanently change the microvascular network, at least in the skin. Already in adolescence, the cIMT is increased independently of high blood pressure values. This may be an expression of early atherogenic susceptibility and could be an important link in understanding the epidemiological associations between low birth weight and increased risk of cardiovascular disease.

Acknowledgements We would like to thank the children and their parents for participating. This work would not have been possible without Helene Kern, who contacted the families.

Contributors HNL and SE collected and analysed the data and wrote the first version of the manuscript; they had full access to all data in the study and take responsibility for the integrity of the data and the accuracy of the data analysis. ZM assisted with study design. RDP was responsible for cardiology data. RDP and OG-B developed and supervised the study, and edited the manuscript.

Competing interests None.

Patient consent Parental/guardian consent obtained.

Ethics approval The Ethics Committee of the Medical Faculty of the University of Munich approved this study.

Provenance and peer review Not commissioned; externally peer reviewed.

\section{REFERENCES}

1 Davies AA, Smith GD, May MT, et al. Association between birth weight and blood pressure is robust, amplifies with age, and may be underestimated. Hypertension 2006;48:431-6.

2 Rossi P, Tauzin L, Grosse C, et al. [Impact of gestational age and birthweight on long-term cardiovascular health]. Rev Med Interne 2007;28:545-51.

3 Goodfellow J, Bellamy MF, Gorman ST, et al. Endothelial function is impaired in fit young adults of low birth weight. Cardiovasc Res 1998:40:600-6.

4 Bonamy AK, Martin H, Jorneskog G, et al. Lower skin capillary density, normal endothelial function and higher blood pressure in children born preterm. $J$ Intern Med 2007;262:635-42

5 Bonamy AK, Norman M, Kaijser M. Being born too small, too early, or both: does it matter for risk of hypertension in the elderly? Am J Hypertens 2008;21:1107-10.

6 Hack M, Schluchter M, Cartar L, et al. Blood pressure among very low birth weight $(<1.5 \mathrm{~kg})$ young adults. Pediatr Res 2005;58:677-84.

7 Hovi $P$, Andersson S, Eriksson JG, et al. Glucose regulation in young adults with very low birth weight. N Engl J Med 2007;356:2053-63.

8 Barker DJ. Fetal nutrition and cardiovascular disease in later life. Br Med Bull 1997:53:96-108
9 Grobbee DE, Bots ML. Carotid artery intima-media thickness as an indicator of generalized atherosclerosis. J Intern Med 1994;236:567-73.

10 Lim SM, Kim HC, Lee HS, et al. Association between blood pressure and carotid intima-media thickness. J Pediatr 2009;154:667-71.

11 Pall D, Settakis G, Katona $E$, et al. Increased common carotid artery intima media thickness in adolescent hypertension: results from the Debrecen Hypertension study. Cerebrovasc Dis 2003:15:167-72.

12 Dalla Pozza R, Bechtold S, Bonfig W, et al. Age of onset of type 1 diabetes in children and carotid intima medial thickness. J Clin Endocrinol Metab 2007:92:2053-7.

13 Jarvisalo MJ, Putto-Laurila A, Jartti L, et al. Carotid artery intima-media thickness in children with type 1 diabetes. Diabetes 2002;51:493-8.

14 Jourdan $C$, Wuhl E, Litwin $\mathrm{M}$, et al. Normative values for intima-media thickness and distensibility of large arteries in healthy adolescents. J Hypertens 2005;23:1707-15

15 Serne EH, Gans RO, ter Maaten JC, et al. Impaired skin capillary recruitment in essential hypertension is caused by both functional and structural capillary rarefaction. Hypertension 2001;38:238-42.

16 Antonios TF, Singer DR, Markandu ND, et al. Rarefaction of skin capillaries in borderline essential hypertension suggests an early structural abnormality. Hypertension 1999:34(4 Pt 1):655-8.

17 Diekmann M, Genzel-Boroviczeny 0, Zoppelli L, et al. Postnatal growth curves for extremely low birth weight infants with early enteral nutrition. Eur J Pediatr 2005; 164:714-23

18 Kromeyer-Hauschild K, Wabitsch M, Kunze D, et al. Perzentile für den Body-mass-Index für das Kindes- und Jugendalter unter Heranziehung verschiedener deutscher Stichproben. Monatsschr Kinderheilkd 2001;194:11.

19 Update on the 1987 Task Force Report on High Blood Pressure in Children and Adolescents: a working group report from the National High Blood Pressure Education Program. National High Blood Pressure Education Program Working Group on Hypertension Control in Children and Adolescents. Pediatrics 1996;98 (4 Pt 1):649-58.

20 Goedhart PT, Khalilzada M, Bezemer R, et al. Sidestream dark field (SDF) imaging: a novel stroboscopic LED ring-based imaging modality for clinical assessment of the microcirculation. Opt Express 2007;15:15101-14.

21 Serne EH, de Jongh RT, Eringa EC, et al. Microvascular dysfunction: a potential pathophysiological role in the metabolic syndrome. Hypertension 2007, 50:204-11

22 Irving RJ, Shore AC, Belton NR, et al. Low birth weight predicts higher blood pressure but not dermal capillary density in two populations. Hypertension 2004:43:610-13.

23 Hiedl S, Schwepcke A, Weber F, et al. Microcirculation in preterm infants: profound effects of patent ductus arteriosus. J Pediatr 2010;156:191-6.

24 Correa M, Andrade L, Kayser C. Comparison of laser Doppler imaging, fingertip lacticemy test, and nailfold capillaroscopy for assessment of digital microcirculation in systemic sclerosis. Arthritis Res Ther 2010;12:R157.

25 Hay WW Jr. Optimizing protein intake in preterm infants. J Perinatol 2009;29:465-6

26 Koletzko B, von Kries $\mathrm{R}$, Closa $\mathrm{R}$, et al. Can infant feeding choices modulate later obesity risk? Am J Clin Nutr 2009;89:1502S-8S.

27 Finken MJ, Inderson A, Van Montfoort N, et al. Lipid profile and carotid intima-media thickness in a prospective cohort of very preterm subjects at age 19 years: effects of early growth and current body composition. Pediatr Res 2006;59(4 Pt 1):604-9.

28 Koletzko B, von Kries R, Monasterolo RC, et al. Infant feeding and later obesity risk. Adv Exp Med Biol 2009:646:15-29.

29 Rabadan-Diehl C, Nathanielsz P. From Mice to Men: research models of developmental programming. J Dev Orig Health Dis 2013;4:3-9. 


\section{ADC In adolescence, extreme prematurity is associated with significant changes in the microvasculature, elevated blood pressure and increased carotid intima-media thickness}

HaNa Lee, Sabrina Dichtl, Zuzanna Mormanova, Robert Dalla Pozza and Orsolya Genzel-Boroviczeny

Arch Dis Child 2014 99: 907-911 originally published online May 30, 2014 doi: 10.1136/archdischild-2013-304074

Updated information and services can be found at:

http://adc.bmj.com/content/99/10/907

These include:

References This article cites 29 articles, 6 of which you can access for free at: http://adc.bmj.com/content/99/10/907\#BIBL

Email alerting Receive free email alerts when new articles cite this article. Sign up in the service box at the top right corner of the online article.

Topic Articles on similar topics can be found in the following collections Collections

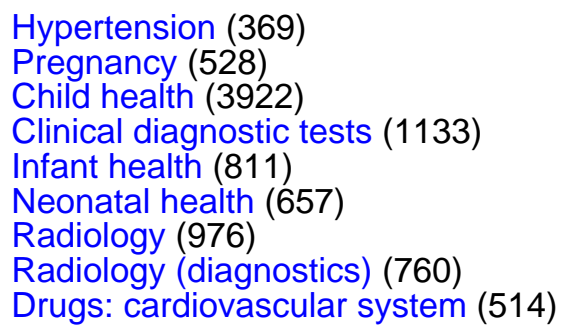

\section{Notes}

To request permissions go to:

http://group.bmj.com/group/rights-licensing/permissions

To order reprints go to:

http://journals.bmj.com/cgi/reprintform

To subscribe to BMJ go to:

http://group.bmj.com/subscribe/ 